\title{
Working Group Progress for IEEE P3141 - Standard for 3D Body Processing, 2017-2018
}

\author{
Carol MCDONALD ${ }^{* 1}$, Alfredo BALLESTER ${ }^{2}$, Randy K RANNOW ${ }^{3}$, \\ Maxim FEDYUKOV ${ }^{4}$, Susan SOKOLOWSKI ${ }^{5}$ \\ ${ }^{1}$ Gneiss Concept, Washougal WA, USA; \\ ${ }^{2}$ Instituto de Biomecánica, Universistat Politècnica de València, Valencia, Spain; \\ ${ }^{3}$ Silverdraft Supercomputing, Boise ID, USA; \\ ${ }^{4}$ Texel Inc., Moskow, Russia; \\ ${ }^{5}$ University of Oregon Portland, Portland OR, USA
}

DOI: 10.15221/18.177 http://dx.doi.org/10.15221/18.177

\begin{abstract}
The 3D Body Processing (3DBP) Industry Connections Working Group, an adjunct group of IEEE P3141, Draft Standard for 3D Body Processing (3DBP), brings together diverse entities devoted to making recommendations for 3D body processing interoperability between creators and consumers of 3D body models. Members are mainly related to the apparel, footwear and accessories industry and include large retailers, scanner providers, data processors, hardware solutions providers, virtual fit providers, small start-ups and universities.
\end{abstract}

This paper summarizes the main activities conducted during the past year and provides an overview of the topics to be addressed in 2019. During 2018, among other activities, the working group published two white papers, determined industry needs with existing file formats, and planned a comparative study of full body using different anthropometric measuring methods, including traditional and digital.

Keywords: 3D Body Processing, Landmarks, Measurements, Anthropometric Measuring, Mass Customization, 3D Scanning, File Formats, Metadata, Digital Last, Comparative Analysis, GDPR, Communication, Security, and Privacy

\section{Introduction}

The IEEE 3DBP Industry Connections Group (IEEE 3DBP) brings together an international, multidisciplinary set of individuals representing many companies that are in the process of proposing new standards and/or practices to enhance 3D body processing interoperability between creators and consumers of 3D body data. The standards will be used in existing industries to develop new businesses around 3D body models.

Companies involved in the 3DBP working group include large retailers, scanner providers, virtual fit providers, CAD tool developers, product manufacturers and small start-ups. The range of body processing involves apparel, footwear and accessories. They impact consumer goods such as apparel, footwear, and wearable accessories. The industry use cases considered thus far range from size recommendation to product personalization, through custom manufacturing, fit predictions and virtual simulation.

Through its members, the working group is connected to other standardization organizations including ISO, X3D, AIST, and ASTM. The group does not intend to duplicate standards and practices but intends to create complementary standards and practices that establish the ecosystem required for future growth of 3D body processing. To help understand the ecosystem's requirements for 3DBP, the working group is closely connected with the 3D Retail Coalition (3DRC) and Product Innovation (PI) association.

The ultimate objective of IEEE 3DBP is to enable the compatible use of digital 3D body data among the different stakeholders (i.e. subjects, data producers, data processors, data brokers and data users), regardless of by whom or where the data was produced. This requires providing recommendations to facilitate an understanding of the 3D object, its values, and data quality retrieved.

The activity of the working group is structured into five sub-groups: File Formats and Metadata; Quality; Communication; Security and Privacy; Footwear; and Mega-Technology Trends. This paper summarizes the main activities conducted from Q4 2017 to Q3 2018 by the working group and provides an overview of the activities planned for 2019.

* Author's Contact: carol@gneissconcept.com 


\section{3DBP Industry Connections Group Current Publications}

In 2017 and 2018, the 3DBP group published a series of white papers, this being the fifth. The white papers serve as introduction to 3DBP technologies guides for future standardization.

White paper \#1: IEEE Industry Connections (IEEE-IC) - 3D Body-model Processing Initiative - An Introduction

As the title [1] suggests, this paper introduced goals for 3D-model body processing (3DBP), especially regarding the need for maintaining interoperability with body models data storage solutions and with the software used in subsequent processing. This white paper is available on the IEEE website

White paper \#2: Working Group Progress for IEEE P3141 - Standard for 3D Body Processing

In October 2107, the IEEE 3DBP published the Working Group Progress for IEEE P3141 - Standard for 3D Body Processing [2] published a summary of activities and progress that the IEEE P3141 Standard for 3D Body Processing had made since Q4 2016, as well as future goals. The white paper summarized the main activities conducted from Q4 2016 to Q3 2017 by the working group and provided an overview of the activities planned for 2018.

White paper \#3: IEEE Industry Connections (IEEE-IC) — Landmarks and Measurement Standards Comparison in 3D Body-model Processing

In February 2018, the Quality subgroup published a second white paper, which was titled IEEE Industry Connections (IEEE-IC) Landmarks and Measurement Standards Comparison in 3D Body-model Processing [3]. This white paper reviews the current standards landscape for three-dimensional body scanning, body landmarking, and measuring. International standards are compared, and recommendations are made for a minimal set of landmarks and measurements (L\&M) needed for several industrial use cases of 3D Body Processing such as fit and size estimation, retail, clothing manufacturing, CAD tool developers, and body model storage and service. This white paper is available on the IEEE 3DBP website.

White paper \#4: IEEE Industry Connections (IEEE-IC) - Personalized Digital Last (a Women's Example) - The Tool Required to Enable Mass Customization

In April 2018, the Footwear subgroup published a white paper titled IEEE Industry Connections

(IEEE-IC) Personalized Digital Last (a Women's Example)—The Tool Required to Enable Mass Customization [4]. This white paper examines the development of a personalized digital last and the impact on the role of footwear technicians. As the personalized digital last becomes the basis of future footwear production, the role of footwear technicians may expand to include a broader definition of fit, maintaining libraries of different lasts, and maintaining quality safeguards for mass customization of footwear. Although this paper focuses on women's footwear, the issue of fit is universal for men, women, and children. The techniques described in this paper can be utilized for most footwear segments. This white paper is available on the IEEE 3DBP website.

\section{Technical Working Subgroups}

Within the 3DBP, there is an umbrella technology working group where separate subgroups are focused in the following five areas:

- File Formats (including Metadata)

- Quality

- Communication, Security and Privacy (CSP)

- Footwear

- Mega Technology Trends

\subsection{File Formats and Metadata Subgroup}

During 2018, this subgroup merged the activities of the former sub groups of Metadata and File Formats due to the overlapping of activities. This working group intends to recommend existing file formats (or to create new ones if needed) that support the linkage and management of 3D data and all its all related metadata. This subgroup also intends to define mandatory and optional metadata derived from 3D processing, the access of which is valuable for industry applications. 
During Q4 2017, the File Formats and Metadata Subgroup conducted a survey across 3D data producers, processors and consumers to determine file format features desired for 3D body processing and extensively evaluated existing 3D model file formats. It resulted in a chart that compares industry requirements with the capabilities of common file formats from which two file formats were short-listed. Results of the survey showed that only some of the features required by 3DBP can be covered by a single file format (Figure 1).

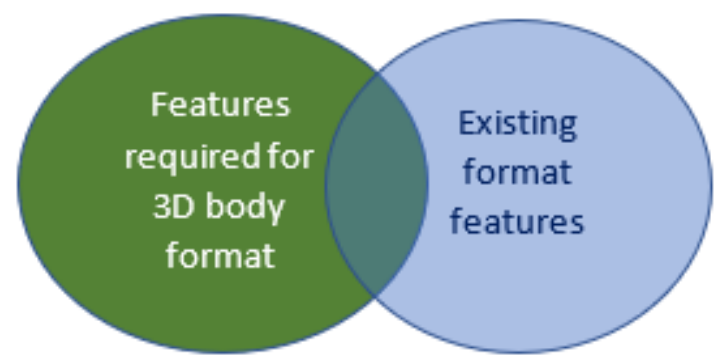

Figure 1. Overlap of 3D body processing and existing formats features

The challenge was to find a good match, such that both sets coincide to the greatest extent possible. Otherwise, two problems arise-if the needed features are not supported in a chosen file format, the human body-related applications get restricted; and if a chosen file format has features that are not needed for 3D body processing, it becomes a source of complexity and incompatibility.

Two possible solutions were considered as follows:

1) To develop a new file format, or

2) To find a good match with required features from existing formats.

The members of the 3D Body Processing Group agreed on the feasibility of the second option.

There are many 3D model file formats available today. Overall, the formats that were explored in detail were: 3DS, 3DXML, 3MF, BLEND, Collada, DSON, DWG, DXF, E57, FBX, gITF, IGES, JT, LWO, MD2, MD3, OBJ, OFF, OpenCTM, OpenGEX, PLY, PRC, STEP, STL, U3D, VRML 1.0, VRML 2.0, X, X3D, $X S I$, and XVL. The File format and Metadata subgroup's main problem was there are existing old file formats which have gained widespread adoption because they are simple, and they are quick to implement in any language. Due to their age, these file formats are not being developed currently, and as such, there is no need to get management approval for future format version adoption. In contrast, the newer, more advanced formats have a rich set of features and extensibility, but they are not widely used.

In summary, the File Formats subgroup lists the choices as the following:

1) Single old file format, e.g., OBJ

2) New advanced file format, e.g., X3D or gITF

3) Something in-between option 1) and option 2); not as advanced and extensible, but widespread, like VRML or FBX

4) The recommendation to use different file formats for different use cases

5) The recommendation to use one file format now (widespread, but not very advanced), and the recommendation to use another file format in the future (when new widespread formats appear)

The File Formats subgroup has concluded that option two, using a recent file format, was the best compromise.

Among these formats, five advanced formats were short-listed: FBX, gITF, X3D (ISO/IEC 19775/19776/19777, successor of VRML), BLEND (Blender native format), DSON (Daz Studio native format). After a thorough analysis and comparison of these formats by the subgroup members and invited experts, the two formats that were determined to be most suitable for 3D Body Processing requirements are X3D and gITF.

During 2019 the subgroup plans to publish a white paper including 3DBP recommendations on file formats to be used and on the use and management of metadata. 
One of the concerns that rose among industry during 2018 is personal data privacy along the 3DBP pipeline, due to the recent release of the European Union (EU) General Data Protection Regulation (GDPR) in May 2018 [5]. Personal data is any data that can be used, using reasonable means, to identify a subject. This European regulation puts emphasis on guaranteeing that each person, as rightful owner of his personal data, should be in control of it. Thus, any personal data and metadata involved in 3DBP pipelines will require explicit permissions with respect to an extensive list which includes recording, storage, transfer, use, depersonalization, and additional data operations. In this regard, this group is already working on identifying among all the data associated with a 3D object what is personal and non-personal data. This will be an important topic of investigation and discussion during 2019.

\subsection{Quality Subgroup}

The Quality sub-group plans to provide methods, tools, benchmarks, resources and testing procedures to define and quantify the quality of 3D models, as well as the quality of the critical metadata for use cases, such as body landmarks and measurements.

In February 2018, this group published a second white paper titled IEEE Industry Connections (IEEE IC) Landmarks and Measurement Standards Comparison in 3D Body model Processing [3], as noted in the Publications section.

The Quality subgroup is preparing a study on Comparative Analysis of Body Measuring Methods to be released in 2019. The results of this study will help apparel companies consuming or using body measurements, to better understand the benefits and limitations of this type of data -- in particular, the reliability of the available methods, as well as the compatibility between same measurements obtained by different methods.

The 2019 study will use volunteers throughout the greater Portland, Oregon area. Due to resource constraints, the study will not be completely random. The sample of subjects will be measured with different anthropometric methods, including traditional and digital. Four apparel industry experts will manually take a series of 11 body measurements via traditional anthropometry repeated three times from every selected subject. Due to time constraints imposed by the study, the list of measurements was narrowed to the 11 identified as being most relevant to the apparel industry. Subjects will also be measured with two different body scanners provided by Human Solutions GmbH (VITUS Bodyscan) and SizeStream, LLC (SS20). Each scanner company will analyze their own data, as well as, the other's. IBV, acting as an independent third party will also analyze the scanner data from both scanner companies. Since digital measuring is much more efficient and is not constrained by time during data gathering, measurements derived from the scanning data will not be limited to the 11 listed measurements taken by traditional anthropometry.

Table 1. List of measurements designations and ISO reference

\begin{tabular}{|l|l|c|}
\hline \multicolumn{1}{|c|}{$\#$} & Designation & ISO 8559-1:2017 [6] \\
\hline 1 & Neck girth & 5.3 .2 \\
\hline 2 & Back neck point to waist & 5.4 .5 \\
\hline 3 & Upper arm girth & 5.3 .16 \\
\hline 4 & Back neck point to wrist & 5.4 .17 \\
\hline 5 & Across back shoulder width & 5.4 .3 \\
\hline 6 & Bust girth & 5.3 .4 \\
\hline 7 & Waist girth & 5.3 .10 \\
\hline 8 & Hip girth & 5.3 .13 \\
\hline 9 & Thigh girth & 5.3 .20 \\
\hline 10 & Total crotch length & 5.4 .18 \\
\hline 11 & Inside leg height (L or R) & 5.1 .15 \\
\hline
\end{tabular}

The sampled subjects will be selected to represent a wide range of female and male body shapes (i.e. heights, weights and body fat percentages). The number of subjects will be optimized to enable independent analyses of data by gender and by body fat percentage (BFP) using the optimal experiment design methods proposed by Walter et al. [7]. The University of Oregon has acted in an advisory role for understanding Institutional Review Board (IRB) requirements and Collaborative Institutional Training Initiative (CITI) [8] requirements for dealing with human subjects. 
The data gathering phase is coordinated by Kalypso and Gneiss Concept and is planned to be held in Oregon (USA) during late 2018. Additional data gathering phases involving other body scanners and measuring experts is planned to be held in 2019.

The analytic procedures planned include the quantification of the reliability of each technique and the reliability of different combinations of techniques (i.e., traditional and digital); and the quantification of the biases between pairs of techniques or pairs of combination of techniques. The results will be communicated in a white paper planned for 2019. The data will be available for review.

Based on the results of this study companies consuming or using body data (e.g. measurements or 3D models) will be able to make an informed use of current methods (traditional and digital) and to effectively integrate 3D data into their design methods. Moreover, this Quality group and other research/technology organizations will be able to use the results and data gathered to investigate and build new standards to aid in resolving existing problems pertinent to reliability and compatibility of measurements with $3 \mathrm{D}$ data.

The objectives and planned outcomes of the study were presented in June 2018 at PI Apparel in New York. This was well received by the brands, since they are aware of the differences in measurements and 3D data and of the lack of compatibility across methods.

\subsection{Communication, Security, and Privacy Subgroup}

The decreasing cost of computing and the increasing availability of wired and wireless connectivity is enabling consumers to generate millions of terabytes of data daily. It is common knowledge of the misuse of millions of Facebook users' personal data by Cambridge Analytica, as well as the theft of the privileged information from the Office of Personnel Management in 2014 where some 22 million federal workers' personal data was compromised. These events led U.S. lawmakers to introduce a flood of privacy-related legislative proposals, with no resulting statues or regulatory changes. This contrasts to the EU, which implemented a sweeping new data privacy law, the GDPR; the U.S. has no comprehensive regulatory entity or agency or no succinct regulatory requirements. Organizations within the U.S. that have interactions with EU citizens and their data must be compliant with GDPR. Those organizations that do not, are not required to comply.

Cisco Visual Networking Index: Forecast and Methodology: 2016-2021 indicates that 82\% of the global network (IP or internet protocol) video traffic will be consumer driven by 2021, and that consumer IP traffic will reach $232.7 \mathrm{~EB}$ per month by 2021. Consumer internet continues to be the main originator of network traffic through 2021, with global consumer network traffic expected to around $233 \mathrm{~EB}$ per month in 2021. Privacy and Security is a program by the Internet Architect Board (IAB) and is designed as a forum as it relates to CSP within the internet technical standards community.

The Communication, Security and Privacy (CSP) subgroup is investigating the secure transmission and storage, as well as the use, protection and privacy of records that contain personal information as it pertains to 3D body processing. The IEEE 3DBP hopes to leverage existing global practices to examine adjacent projects that may provide insights into CSP procedures, establish and formalize processes to help ensure CSP methods, and develop minimum CSP practices to improve individual privacy and security, regardless of the communication protocol used in information or record exchange and storage. This includes working with the IETF as they continue to work on the DNS privacy solution. Additionally, RFC 7721 (Security and Privacy Considerations for IPv6 Address Mechanisms) examines privacy and security considerations for IPv6 addressing and threat mitigation.

Internet connected 3DBP devices may be a subset of the Internet of Things (IoT) and big data, and the CSP is working to ensure specific sensitivity for consumers and users. If 3DBP devices or their information is compromised, the sensitive or personal data information may be used for non-authorized or illegal activities. Thus, the CSP subgroup is evaluating practices and procedures necessary to establish and maintain reasonable procedures to protect the confidentiality, security, and integrity of personal information that may be collected or used with 3DBP.

All communications shared between 3DBP equipment may be transmitted via unencrypted and unauthenticated HTTP GET and POST requests. Communications include sharing of static and dynamic content, thus potentially enabling a spoof event with the arbitrary 3DBP content. One preventative approach is to ensure all personal or private information is behind secured networks that have controlled access, and that information is encrypted via Secure Socket Layer (SSL) technology. 
To fully examine and address 3DBP data and information, CSP is surveying and evaluating the physical and network layers in terms of authentication, access control, confidentiality, integrity and availability to help ensure minimal risk.

Going forward, restrictions on 3DBP information use or transmission shall occupy a central place in CSP framework. This may include the use of technology (e.g. machine learning/ deep learning) to assist with practices and enforcement of 3DBP information. The CSP subgroup will leverage use, to extent possible, practices and procedures as it relates to 3DBP data integrity. The CSP subgroup will coalesce existing standards information and review for completeness relative to 3DBP data. The subgroup will do a gap analysis and drive new initiatives to ensure interoperability, as well as data integrity and confidentiality.

\subsection{Footwear Subgroup}

The Footwear subgroup was formed to address how the different concerns of footwear can impact customer 3D scanning data.

Footwear sizing concerns are as follows:

- Footwear dimensions are impacted by the amount of weight on the foot

- Footwear is last based-either physical or digital

- There is a lack of consensus as to what constitutes critical measurements for fit in footwear

- There is a lack of understanding of how compression of soft tissue (the amount of soft tissue / "boniness" the foot and the perception of fit by the consumer) affects fit in footwear

In addition, footwear design software is very expensive relative to apparel software. The apparel software generally costs around \$2400 USD per year (subscription basis), whereas dedicated footwear software costs in the range of $\$ 13,000$ to $\$ 30,000$ USD per license depending on the number of software modules purchased.

The Footwear subgroup presented the paper noted previously at UITIC International Technical Footwear Congress in May 2018. This allowed for industry exposure to the future of mass customization and the impact to the footwear industry.

The Footwear Subgroup is looking to understand the dynamics of customer fit with the focus on predicative indicators and soft tissue research. Predicative indicators allow for customer perception of fit along with customer foot scans or avatars. Together these aid the Virtual Fitting Algorithms (VFA) or Artificial Intelligence (Al) or Best Fit between lasts or Temporarily Modified Lasts for customer's designated fit. Additional research will be focused on foot soft tissue as it relates to the understanding of foot scans.

\subsection{Mega Technology Trends Subgroup}

The Mega Technology Trends subgroup plans to analyze how new technologies based on artificial intelligence (Al), cloud computing, Internet of Things, big data, 5G communications, and other technologies may impact the retail environment.

There are many theories on information technology and impact on society. However, because of varying cultural norms, there is mostly likely not a single action that the Mega Technology Subgroup can provide as an all-encompassing solution. Today, people live in an information society where data and communications play an ever-evolving and constantly expanding role in daily life. The subgroup will explore the sociological issues that may be experienced with or related to 3DBP, and will help ensure a shared vision of understanding of how the technology and its use may be better understood by consumers. The 3DBP endeavor will reach out to the extent possible, to adjacent standards bodies, as well as industry practitioners to help facilitate a shared understanding of the technology and its deployment.

\section{Conclusions}

The vision and goal of the 3DBP initiative is to create complementary standards and practices that promote an ecosystem that "lifts all boats" and drives future growth opportunities for players across the 3D body processing value chain. On-going activities of the IEEE 3DBP working group will establish recommendations (as documented in white papers) for: an interoperable 3D file format, data quality, meta-data, protocols, and data security (including privacy concerns). 
Phase One of the Comparative Analysis of Measuring Methods will be completed and reported upon. Collaboration with other standards committees and organizations is integral to the goals of this committee, and industry participation is critical to ensure current and future standardization needs of this expanding industry are addressed. Interested parties are invited to contact IEEE 3DBP for further information, https://standards.ieee.org/industry-connections/3d/bodyprocessing.html

\section{References}

[1] Stahl, Michael. (February 2017) "IEEE Industry Connections (IEEE-IC)—3D Body-model Processing Initiative-An Introduction," New York, IEEE, PDF: ISBN 978-1-5044-3780-6, https://standards.ieee.org/develop/indconn/3d/bodyprocessing.html.

[2] McDonald, Carol; Oviedo, Luciano; Ballester, Alfredo. "Working Group Progress for IEEE P3141 Standard for 3D Body Processing"

Proceedings of 3DBODY.TECH 2017 8th International Conference and Exhibition on 3D Body

Scanning and Processing Technologies, Montreal, Canada, 11-12 Oct. 2017, PDF,

DOI:10.15221/17.328, http://dx.doi.org/10.15221/17.328

[3] McDonald, Carol; Wu, Yingying; Ballester, Alfredo; Stahl, Michael. (February 2018) "IEEE Industry Connections (IEEE-IC) Landmarks and Measurement Standards Comparison in 3D Body-model Processing," New York, IEEE, PDF: ISBN 978-1-50444655-6, https://standards.ieee.org/develop/indconn/3d/bodyprocessing.html.

[4] McDonald, Carol; Golub, Andrey. (April 2018) "IEEE Industry Connections (IEEE-IC) Personalized Digital Last (a Women's Example)-The Tool Required to Enable Mass Customization," New York, IEEE, PDF: ISBN 978-1-5044- 4860-1, https://standards.ieee.org/develop/indconn/3d/bodyprocessing.html.

[5] Regulation (EU) 2016/679 (General Data Protection Regulation) in the current version of the OJ L 119, 04.05.2016; cor. OJ L 127, 23.5.2018, (GDPR), https://gdpr-info.eu/

[6] ISO 8559-1:2017, Size designation of clothes - Part 1: Anthropometric definitions for body measurement, https://www.iso.org/standard/61686.html

[7] Walter, S.D. Dr., Eliasziw, M., Donner, A., "Sample size and optimal designs for reliability studies", Dec 1998,John Wiley \& Sons, Ltd, https://doi.org/10.1002/(SICI)10970258(19980115)17:1<101::AID-SIM727>3.0.CO;2-E

[8] Collaborative Institutional Training Initiative (CITI program), Social-BehavioralEducational (SBE) Basic, https://about.citiprogram.org/en/course/human-subjectsresearch-2/. 